Check for updates

Cite this: RSC Adv., 2018, 8, 28934

\title{
Preparation of triangular silver nanoplates by silver seeds capped with citrate-CTA ${ }^{+} \uparrow$
}

\author{
Yuanzhi Zhong, (D) + Guorun Liang, (D) $\$$ Wenxiu Jin, (D) Zhichao Jian, \\ Zhixiong Wu, (D) Qingyuan Chen, (D) Yuchun Cai (D) and Wanzhong Zhang (D)*
}

Due to the competitive growth on the crystal face of seed, it is always difficult to control the morphology of the formation of nanoparticles precisely by a seed-mediated growth method. Herein, we provided a simple but effective technique to synthesize silver nanotriangles using a new silver seed that is capped with citrate-CTA ${ }^{+}$CTA $^{+}$is cetyltrimethyl ammonium cation). Compared to the preparation of silver nanoparticles (AgNPs) by a conventional seed-mediated method, in this paper, we presented a growth technique with two distinct innovative changes. First, the concentrations of CTAB that we added in silver seed collosol have a significant impact on the size distribution, and silver nanotriangles, nanorods, and nanospheres could be obtained by adjusting the CTAB concentration. Second, the seed prepared by our method has a longer use time, and silver nanotriangles, nanospheres, and nanorods could be prepared by adjusting the aged time of the seed colloid. We have also shown a simple way to control the morphology of silver nanoparticles in almost the same reactive medium by varying the $\mathrm{NaOH}$ concentration. Using the new silver seed capped with citrate-CTA ${ }^{+}$, we obtained triangular silver nanoparticles with relatively high regularity. Based on the limited experimental results and IR analysis, a possible mechanism was preliminarily proposed to explain the formation of the seed and the truncated triangular AgNPs.

Received 28th May 2018

Accepted 3rd August 2018

DOI: $10.1039 / c 8 r a 04554 b$

rsc.li/rsc-advances have been evidenced that triangular silver nanoplates have a promising application to selectively detect some kinds of volatile compounds, e.g. chlorides. ${ }^{27,28}$ Compared to Ag nanoparticles and $\mathrm{Ag}$ bulk materials, triangular silver nanoplates have significant catalytic properties in the shape-dependent electrocatalytic reduction of $\mathrm{CO}_{2}$ to $\mathrm{CO}^{29}$

Nowadays, a variety of synthesis methods have been used to prepare triangular silver nanoplates, including photochemical route, seed-mediated route, electrochemical route, thermal induction route and sonochemical route. ${ }^{30,31}$ In 2001, the photochemical route was first introduced by Mirkin $e t$ al. as an effective synthesis method for triangular silver nanoplates. ${ }^{32}$ However, it requires light sources for excitation of the reaction system, including ion laser lines, light-emitting diodes (LEDs), ultraviolet (UV) lamp and sodium lamp, ${ }^{33-37}$ which are generally more expensive and delicate. Subsequently, Murphy et al. first reported a seed-mediated method to study the kinetic control of the formation of triangular silver nanoplates,$^{38}$ which had a profound impact on the subsequent studies. Due to the competitive growth on the crystal faces of seed, it is always difficult to precisely control the morphology of the formed nanoparticles. Tang et al. reported triangular silver nanoplates that were synthesized using sunlight as the photoconductive source ${ }^{36}$ However, it is difficult to control the natural sunlight, particularly lighting power, which may vary every day. In addition, the method is facile but inefficient due to the long time irradiation.
Guangdong Provincial Key Laboratory of New Drug Screening, School of Pharmaceutical Sciences, Southern Medical University, Guangzhou 510515, PR China. E-mail: zhangwz6@smu.edu.cn; Tel: +86 2062789465

$\dagger$ Electronic supplementary information (ESI) available. See DOI: 10.1039/c8ra04554b

$\ddagger$ These authors contributed equally to this work. 
In the seed-mediated system, first, a metal precursor (e.g., $\mathrm{AgNO}_{3}$ ) is generally reduced by a strong reducing agent (e.g., $\mathrm{KBH}_{4}$ ) to synthetize seed crystals. Then, a growth process of the seed occurred with the reduction of $\mathrm{AgNO}_{3}$ by a weak reducing agent (e.g., ascorbic acid (Vc)) in the solution containing the seed crystals and cetyltrimethyl ammonium bromide (CTAB). ${ }^{39}$ We noticed that gold nanorods were synthesized by an improved seed-mediated method to obtain the nanorods with high regularity. ${ }^{\mathbf{4 0 , 4 1}}$ In their experiment, CTAB was added for the formation of gold seed to form a CTAB-capped seed instead of a citrate-capped seed.

Herein, we provide a simple but effective technique to synthesize silver nanotriangles using silver seeds capped with citrate-CTA ${ }^{+}$. To the best of our knowledge, only a few reports focused on silver seeds capped with citrate-CTA ${ }^{+}\left(\mathrm{CTA}^{+}\right.$is cetyltrimethyl ammonium cation). We finally obtained triangular silver nanoplates instead of silver nanorods, which were usually reported in published literature using a typical seedmediated growth method..$^{38}$

To overcome the disadvantages in the preparation of AgNPs (e.g., high polydispersity, that is, the formation of silver nanorods or triangular silver nanoplates accompanying spherical and quasispherical nanoparticles), we attempted to improve the preparation method of silver seeds by adding CTAB in this process. Surprisingly, the addition of CTAB in silver seed had a marked impact on the morphology of final AgNPs. Another interesting finding in this study was that the seed prepared by this method had a longer use time, and the aged time of the seed colloid can control the morphology of the formed nanoparticles. At last, we propose a possible mechanism for the formation of silver seeds and triangular silver nanoplates based on some experimental results and IR analysis.

\section{Experimental section}

\subsection{Experimental materials}

Silver nitrate $\left(\mathrm{AgNO}_{3}, \mathrm{AR}\right)$, cetyltrimethyl ammonium bromide (CTAB, Amresco 0833, AR), trisodium citrate (AR), ascorbic acid (Vc, AR), sodium hydroxide ( $\mathrm{NaOH}, \mathrm{AR}), \mathrm{KBH}_{4}$ (AR, dissolved in cold (ice-bath) aqueous solution of $\mathrm{NaOH}(0.01 \mathrm{M})$ ), and sodium bromide ( $\mathrm{NaBr}, \mathrm{AR})$ were used in this study. Water used in the experiments was double-distilled water.

\subsection{Preparation of silver seed colloidal solution}

Typically, $0.2 \mathrm{~mL}$ of $0.1 \mathrm{M}$ CTAB, $0.5 \mathrm{~mL}$ of $0.01 \mathrm{M} \mathrm{AgNO}_{3}$ and $0.5 \mathrm{~mL}$ of $0.01 \mathrm{M}$ sodium citrate were added into $19 \mathrm{~mL}$ doubledistilled water, in sequence, and stirred vigorously for a minute (except for the Section 3.1). Then, $0.6 \mathrm{~mL}$ of $0.01 \mathrm{M} \mathrm{KBH}_{4}$ was added to the mixed solution and stirred slightly until the colloidal solution changed from bright yellow to green yellow, indicating the formation of small silver nanocrystals. The temperature was maintained at $28{ }^{\circ} \mathrm{C}$. The silver nanocrystals dispersed in the colloidal solution were used as silver seeds in different times (from 0 up to $24 \mathrm{~h}$ ) after preparation. In this case, the concentration of CTAB was $0.96 \mathrm{mM}$ for the formation of the seeds. For CTAB solution, the first critical micelle concentration of $\mathrm{CTAB}\left(\mathrm{CMC}_{1}\right)$ was $0.84 \mathrm{mM}$, while the second critical micelle concentration of CTAB $\left(\mathrm{CMC}_{2}\right)$ was $7 \mathrm{mM} \cdot{ }^{42}$ The concentration of $\mathrm{CTAB}$ in the seed colloidal solution was between $\mathrm{CMC}_{1}$ and $\mathrm{CMC}_{2}$. Therefore, the seed colloidal solution with CTAB formed spherical micelles.

\subsection{Preparation of AgNPs with different morphologies}

Typically, $15 \mathrm{~mL}$ of $0.1 \mathrm{M} \mathrm{CTAB}$ and $0.5 \mathrm{~mL}$ of $0.01 \mathrm{M} \mathrm{AgNO}_{3}$ were put into a $50 \mathrm{~mL}$ clean and dried conical flask, in sequence, and stirred slightly. Then, $0.25 \mathrm{~mL}$ of the seed colloidal solution and $1 \mathrm{~mL}$ of $0.1 \mathrm{M} \mathrm{Vc}$ and $3 \mathrm{~mL}$ of $0.1 \mathrm{M} \mathrm{NaOH}$ were injected into the mixture, in sequence (except for Section 3.3). The color of the colloidal solution turned yellow, red, purple, and finally blue within 3 minutes under vigorous stirring. The colloidal solution will be called as growth solution in the following discussion. The concentration of CTAB in the solution was $76 \mathrm{mM}$, which was over $\mathrm{CMC}_{2}$, indicating that CTAB molecules formed rod-like micelles in the growth system.

\subsection{Separation and characterization}

Initially, $9 \mathrm{~mL}$ of the as-prepared AgNPs collosol was centrifuged at $6000 \mathrm{rpm}$ for $30 \mathrm{~min}$ and the obtained precipitate was redispersed in $8 \mathrm{~mL}$ of double-distilled water to centrifuge at the same conditions. Then, the second supernatant taken from above collosol was centrifuged at $10000 \mathrm{rpm}$ for $10 \mathrm{~min}$. The obtained precipitate was dispersed in $0.2 \mathrm{~mL}$ of double-distilled water to prepare samples for TEM test. TEM images of the asprepared AgNPs were acquired using a JEM-2100HR transmission electron microscope. UV-vis spectra of the samples were recorded using a UV-3900 spectrophotometer and the hydrodynamic sizes of the samples were measured on a Zetasizer Nano ZS90 (Malvern Instruments, Malvern, U.K.).

\section{Results and discussion}

\subsection{The influence of CTAB on the formation of the seed and AgNPs}

The UV-vis spectra can reflect the shapes and sizes of the obtained AgNPs. ${ }^{18,19,32,43-45}$ For the plasmon resonance absorption of spherical AgNPs, the maximum absorption wavelength $\left(\lambda_{\max }\right)$ is related to the average size of the nanoparticles. The $\lambda_{\max }$ will red shift with an increase in the average size of AgNPs. ${ }^{17-19,43}$ Fig. 1 shows the influence of different concentrations of CTAB in the seed on the formation of silver seed and AgNPs. Clearly, the $\lambda_{\max }$ of the absorption peaks of the seed collosol located at around $389 \mathrm{~nm}$ without adding CTAB into the seed solution and the corresponding UV-vis spectra of growth solution prepared by the seeds had two typical absorption peaks corresponding to silver nanorods. ${ }^{\mathbf{4 6 4} 7}$ This result was consistent with that of the typical preparation method of silver nanorods published elsewhere. ${ }^{38}$

However, the reaction mixtures of the two seeds were almost colorless but with slightly dark color (Fig. S2 $\dagger$ ), and no absorption peaks appeared when the concentrations of CTAB were 0.48 and $0.72 \mathrm{mM}$. Apparently, these two kinds of seeds were unstable and aggregated into large particles that without 

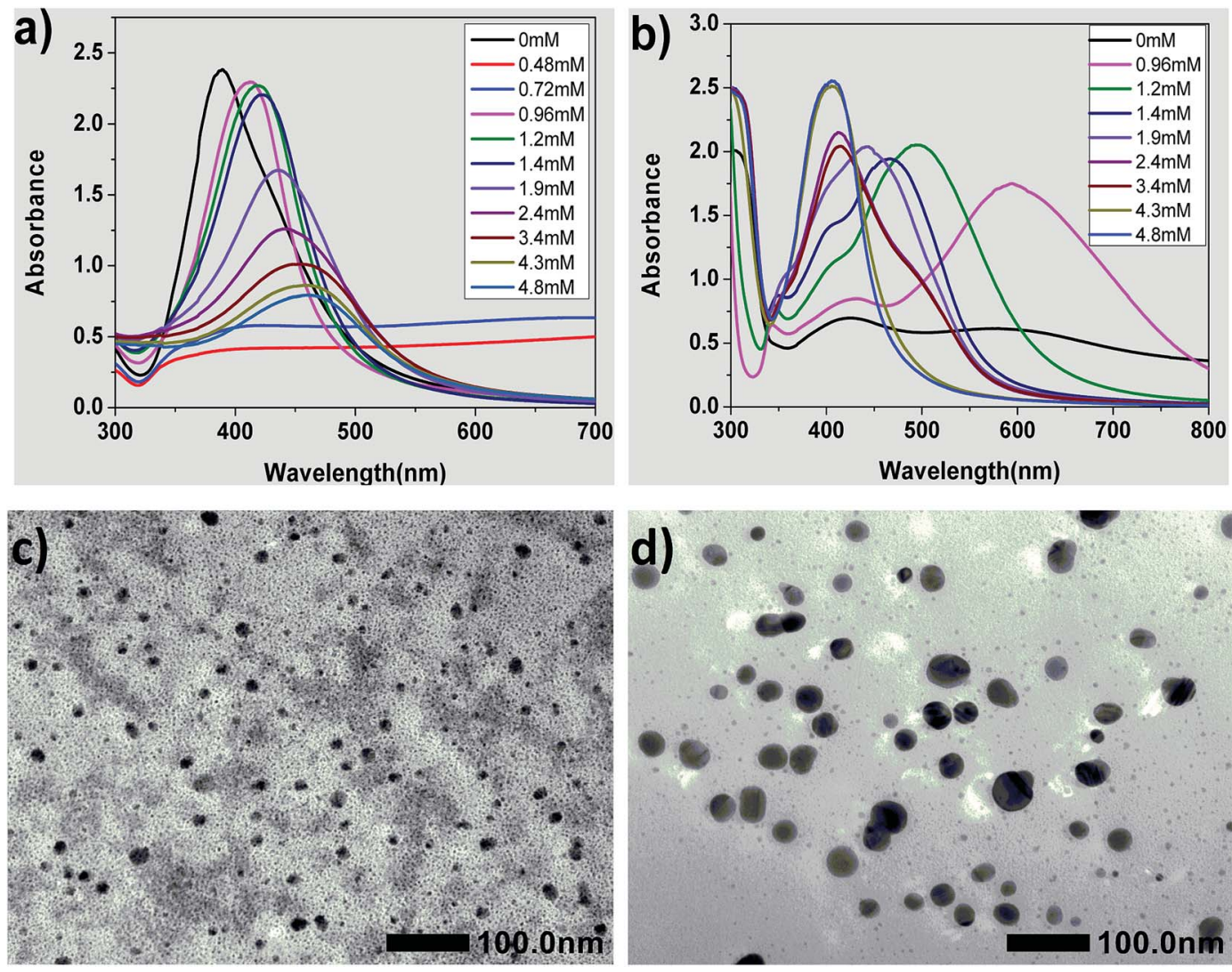

Fig. 1 The influence of different concentrations of CTAB on the formation of the seed and AgNPs. (a) UV-vis spectra of the seed added different concentrations of CTAB. (b) UV-vis spectra of growth solution prepared by the seed that was added different concentrations of CTAB and aged for $20 \mathrm{~min}$. (c) TEM image of silver seeds prepared by adding $0.96 \mathrm{mM}$ CTAB. (d) TEM image of silver seeds prepared by adding $1.90 \mathrm{mM}$ CTAB.

plasmon resonance absorption. Therefore, the seeds obtained using 0.48 and $0.72 \mathrm{mM} \mathrm{CTAB}$ could not be used. We thought that citrate anion served as stabilizer at $0 \mathrm{mM} \mathrm{CTAB}$ in the seed solution, and the seed should be grown to silver nanorods, which was the same as the typical seed growth method. When the concentrations of CTAB were low in the seed solution, i.e., more than $0 \mathrm{mM}$ but less than $0.72 \mathrm{mM}$, the electric potential that exerted an influence on the stability of the seed colloidal solution was counteracted by $\mathrm{CTA}^{+}$due to its opposite charge to citrate. To verify this deduction, the electric potential of the seed colloidal solutions were tested, and the results showed that their potentials were all about $0 \mathrm{mV}$.

As shown in Fig. 1(a), the $\lambda_{\max }$ of the absorption peaks of the seed collosol are located at around 412, 417, 422, 435, 441, 454, 458 and $462 \mathrm{~nm}$ when the concentrations of CTAB changed from 0.96 to $4.80 \mathrm{mM}$. Clearly, the $\lambda_{\max }$ of the absorption peaks red-shifted with an increase in the concentrations of CTAB added to the seed collosol. These results indicate that the average size of silver seeds increased with an increase in CTAB concentration (from 0.96 to $4.80 \mathrm{mM}$ ).

Fig. 1(c) and (d) show the TEM images of silver seeds prepared by adding $0.96 \mathrm{mM}$ CTAB and $1.90 \mathrm{mM}$ CTAB, respectively. The silver seeds were nearly spherical in shape, which was consistent with the single absorption peak observed in the absorption spectrum of silver nanospheres. As shown in the images, the average size of the seeds is $14 \pm 4 \mathrm{~nm}$ (Fig. 1(c)) and $31 \pm 8 \mathrm{~nm}$ (Fig. 1(d)). These results further confirm that the average size of silver seeds increases with an increase in CTAB concentration (from 0.96 to $4.80 \mathrm{mM}$ ).

Fig. 1(b) shows the UV-vis spectra of growth solution prepared by the seed with various concentrations of CTAB. While using the seed prepared by adding $0.96 \mathrm{mM} \mathrm{CTAB}$, the growth solution has three absorption peaks centered at about 343, 431 and $594 \mathrm{~nm}$, which imply that the AgNPs were triangular silver nanoplates. ${ }^{32,44,45}$ On increasing the CTAB concentration to 1.20 and $1.40 \mathrm{mM}$, three absorption peaks appear in the UV-vis spectra, but the $\lambda_{\max }$ is significantly blue shifted, implying that the AgNPs were still triangular silver nanoplates but the average side length is smaller than that in the case of $0.96 \mathrm{mM}^{32,44,45}$ Only spherical nanoparticles were formed in the cases of high concentration of CTAB (i.e., over $4.30 \mathrm{mM}$ ). ${ }^{18,19,43}$ These results imply that triangular silver nanoplates were prepared using the seed by adding CTAB from 0.96 to $1.40 \mathrm{mM}$ and the concentration of СТAB in the seed colloidal solution plays an important role in controlling the size and morphology of AgNPs.

\subsection{The influence of the aging time of silver seed on the morphology and size of AgNPs}

Fig. 2 shows the influence of the aging time of silver seed on the morphology and size of AgNPs. Fig. 2(a) represents the UV-vis spectra of seed colloidal solution at different aging times. The 

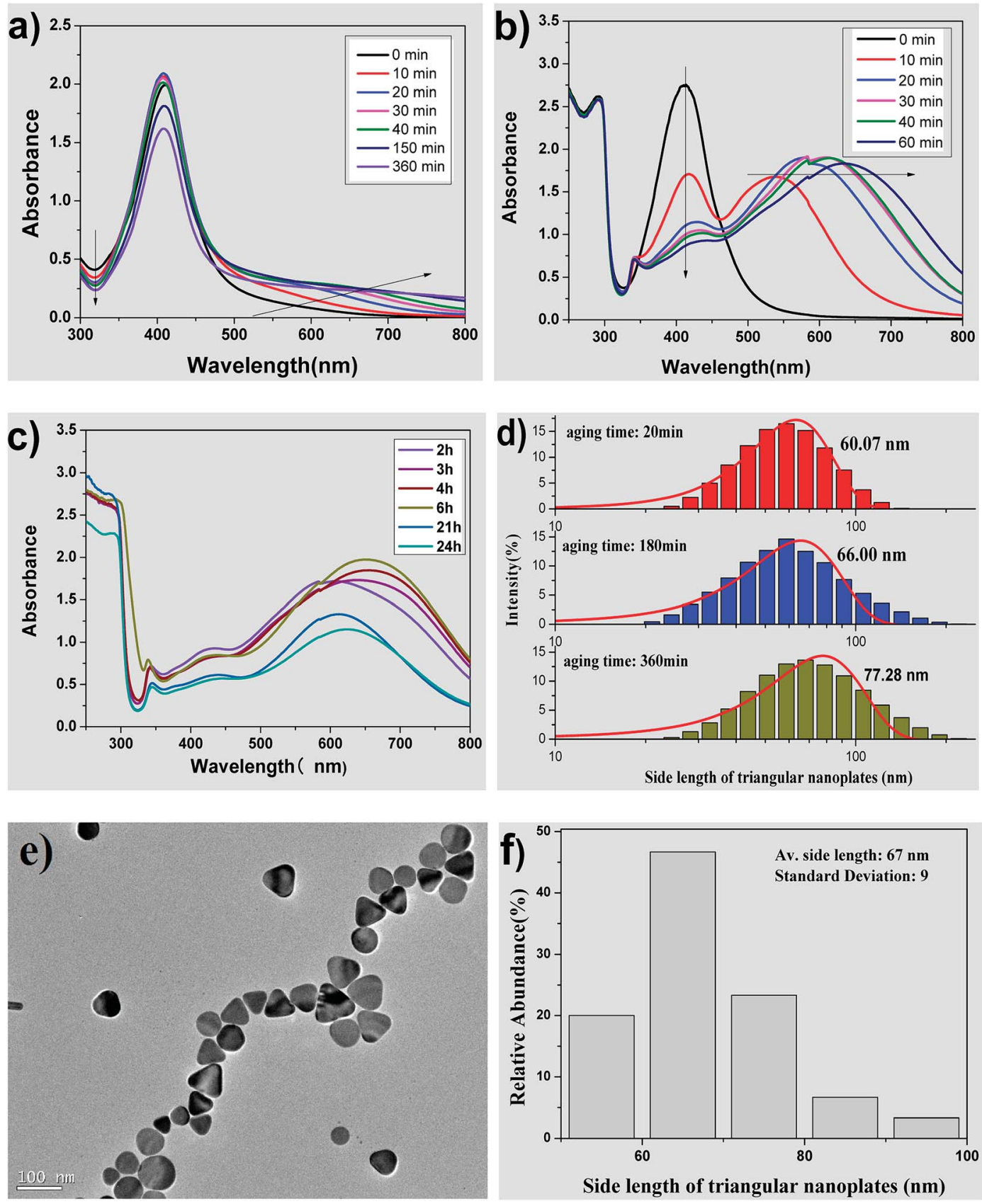

Fig. 2 The influence of the aging time of silver seed on the morphology and size of AgNPs: (a) UV-vis spectra of the seed colloidal solution at different aging times; (b) and (c) UV-vis spectra of growth solution prepared by the seed with the aging times from 0 to 60 min and 2 to 24 h; (d) the hydrodynamic size distribution (by DLS) of silver nanoplates prepared by the seed with different aging times; (e) and (f) TEM image and size distribution of triangular silver nanoplates prepared by the seed aged for $3 \mathrm{~h}$.

absorbance intensity of the absorption peaks at about $410 \mathrm{~nm}$ increase with time within $20 \mathrm{~min}$ and then decrease slightly in the interval from 20 to $40 \mathrm{~min}$. Moreover, the full width at half maximum (FWHM) showed almost no significant change at this time interval. These spectral phenomena imply that silver seeds can be easily prepared and can remain relatively stable in terms of particle size. When the aging time increases over $150 \mathrm{~min}$, the absorbance intensity at about $410 \mathrm{~nm}$ decreases significantly with time. It was reported that the decrease in intensity resulted in the aggregation of silver seed to form crystal membrane; thus, the seed was not used after $5 \mathrm{~h}$ for the preparation. ${ }^{38}$ The marked difference, however, is that the seed prepared by our improved method could be used for preparing triangular silver nanoplates even if the seed is aged for over $24 \mathrm{~h}$.

What happened to the seed in their ageing interval? When the seed was prepared by a conventional method without CTAB, the absorption at about $320 \mathrm{~nm}$ decreased with time, but the absorption at wavelengths over $600 \mathrm{~nm}$ was very low. Due to the selective adsorption of citrate on the special crystal face and orientation effect of rod-like micelles, silver seeds grew 
controllably to form silver nanorods. ${ }^{38}$ Compared to a decrease in absorption intensity at about $320 \mathrm{~nm}$, the absorption at wavelengths over $600 \mathrm{~nm}$ increased with the aging time of seed, which might be caused by the competitive adsorption between citrate and CTAB added into the seed collosol in our case.

Fig. 2(b) and (c) show the UV-vis absorption spectra of growth solution prepared by the seeds with different aging times. When the aging time of the seed is $0 \mathrm{~min}$, the corresponding UV-vis spectrum has only one absorption peak at about $410 \mathrm{~nm}$ with relatively narrow FWHM, indicating the formation of silver nanospheres..$^{\mathbf{1 8 , 1 9 , 4 3 , 4 6}}$ When the aging time is $10 \mathrm{~min}$, two typical absorption peaks appear at about $410 \mathrm{~nm}$ and $530 \mathrm{~nm}$, representing the formation of typical silver nanorods. ${ }^{47,48}$ When the aging time of the seed exceeds $20 \mathrm{~min}$, three absorption peaks appear at about 340, 410 and over $580 \mathrm{~nm}$, respectively, indicating the formation of the triangular silver nanoplates. ${ }^{\text {32,44,45 }}$ Furthermore, the absorbance intensity at about $410 \mathrm{~nm}$ decreases and the $\lambda_{\max }$ at long-wave direction redshifted with aging time from $20 \mathrm{~min}$ to $6 \mathrm{~h}$. These results imply that the average side length of triangular silver nanoplates become increasingly larger with aging time of the silver seeds. ${ }^{32,44,45}$ When the aging time was over $21 \mathrm{~h}$, the absorption peak still had three absorption peaks, although the $\lambda_{\max }$ at longwave direction red-shifted and the absorption intensity decreased with aging time. In other words, the silver nanocrystals prepared by adding CTAB in our case could be used as a seed to prepare triangular silver nanoplates even if the aging time was increased to $24 \mathrm{~h}$.

Fig. 2(d) shows the hydrodynamic sizes measured via dynamic light scattering (DLS). The side lengths of triangular nanoplates form a normal distribution and increases with aging times of the seeds. The average side lengths are $60.07,66.00$ and $77.28 \mathrm{~nm}$, respectively, while the seed was aged for 20, 180 and $360 \mathrm{~min}$. TEM image and size distribution of triangular silver nanoplates prepared by the seed aged for $3 \mathrm{~h}$ are shown in Fig. 2(e) and (f). Apparently, most of the nanoparticles are perfectly truncated triangular nanoplates, as observed in the TEM image. The side length of the nanoplates are mostly between 50 to $80 \mathrm{~nm}$. The average side length of the triangular silver nanoplates id $67 \pm 9 \mathrm{~nm}$, which is slightly different from the value measured via DLS using the seed aged for $180 \mathrm{~min}$.

\subsection{The influence of pH on the morphology of AgNPs}

Fig. 3(a) and (b) show the UV-vis absorption spectra of AgNPs prepared using various concentrations of $\mathrm{NaOH}$. There is no absorption peak in the UV-vis spectrum on adding $5.1 \mathrm{mM} \mathrm{NaOH}$ into the growth solution, indicating that no silver nanoparticles were formed in the solution. When the concentration of $\mathrm{NaOH}$ was adjusted from 8.9 to $15 \mathrm{mM}$, three absorption peaks $(340,410$
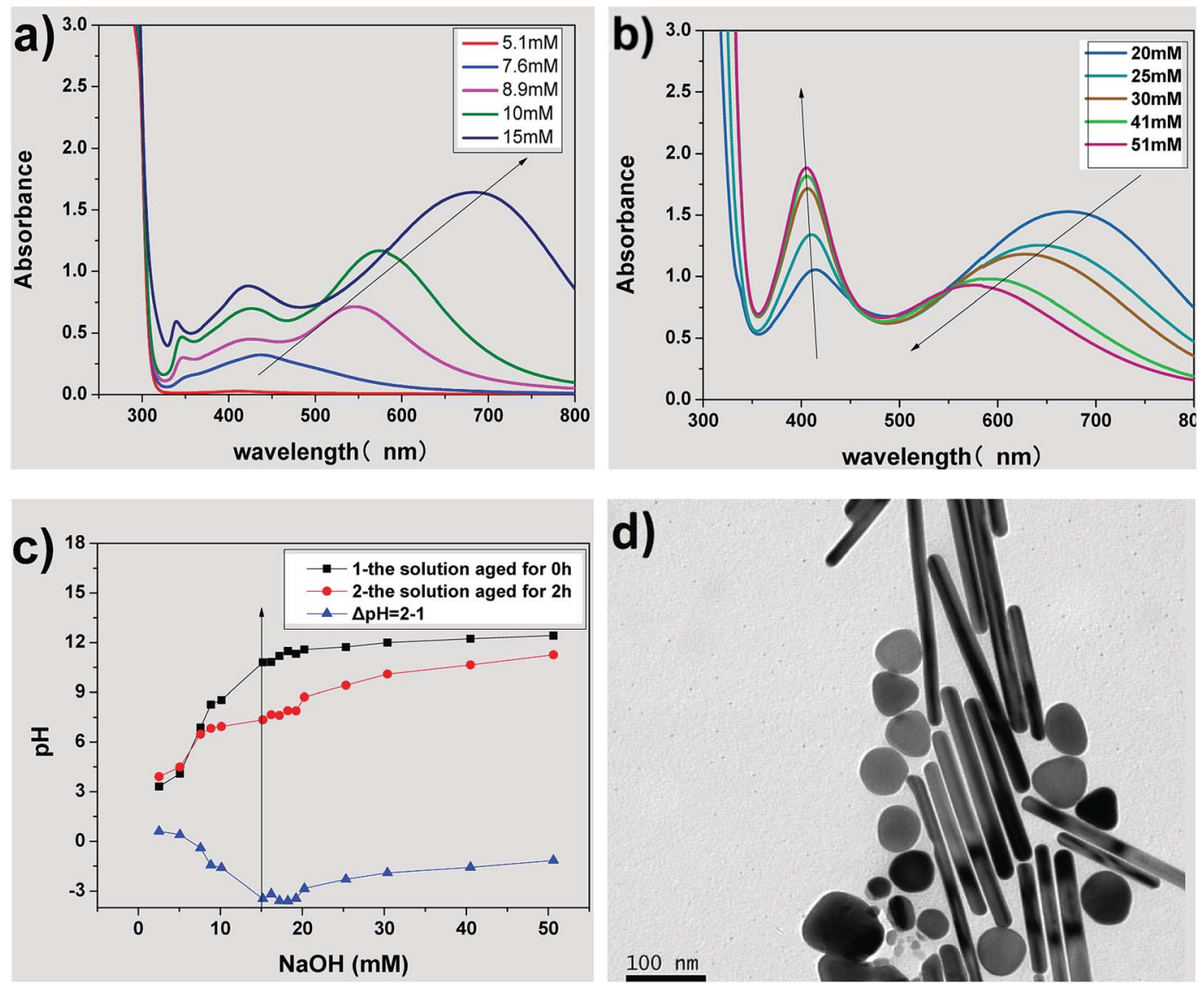

Fig. 3 The influence of the concentrations of $\mathrm{NaOH}$ on the morphology of AgNPs: UV spectra of growth solution with different concentrations of $\mathrm{NaOH}$, (a) $5.1 \mathrm{mM}$ to $15 \mathrm{mM}$, (b) $20 \mathrm{mM}$ to $51 \mathrm{mM}$; (c) the $\mathrm{pH}$ of the growth solution with different concentrations of $\mathrm{NaOH}$. (d) TEM image of nanorods prepared by the growth solution of $30 \mathrm{mM} \mathrm{NaOH}$. 
and over $550 \mathrm{~nm}$ ) appear, as shown in Fig. 3(a), and the $\lambda_{\max }$ of the absorption peak at the long-wave direction had red-shifted, indicating the formation of the triangular silver nanoplates. In addition, their average side length increased with $\mathrm{NaOH}$ concentration. ${ }^{32,44,45}$ When the concentration of $\mathrm{NaOH}$ was adjusted from 20 to $51 \mathrm{mM}$, two absorption peaks appeared at about $410 \mathrm{~nm}$ and over $550 \mathrm{~nm}$ (as shown in Fig. 3(b)), indicating the formation of silver nanorods. Furthermore, the $\lambda_{\max }$ of the absorption peak in the long-wave direction had a blue shift along with a decrease in absorption intensity. Moreover, the absorbance intensity of the peaks at about $410 \mathrm{~nm}$ continuously increased, indicating the average aspect ratio of silver nanorods decreased with an increase in $\mathrm{NaOH}$ concentration. ${ }^{47,49}$ Fig. 3(d) shows the TEM image of nanorods prepared by the growth solution of $30 \mathrm{mM} \mathrm{NaOH}$. As shown in the image, the nanoparticles were mainly nanorods (about $57 \%$ in the image) along with some nanoplates (about 14\%) and some nanospheres (nearly 29\%), which further confirmed our discussions about the TEM image in Fig. 3(b). Clearly, the concentrations of $\mathrm{NaOH}$, i.e., the $\mathrm{pH}$ value of the growth solution, have significant influence on the morphology of AgNPs.

So what factor contributed to the changes in the growth solution of different concentration of $\mathrm{NaOH}$ ? When adding silver seed, $\mathrm{Vc}$ and $\mathrm{NaOH}$ into the growth solution under vigorous stirring, $\mathrm{AgBr}$ was reduced to $\mathrm{Ag}^{0}$ by $\mathrm{Vc}$ (reaction (1)). According to reaction (1), the $\mathrm{pH}$ value (the concentration of $\mathrm{NaOH}$ ) of the growth solution would decrease with the progress of the reduction reaction.

$$
2 \mathrm{OH}^{-}+2 \mathrm{AgBr} \stackrel{\mathrm{Vc}}{\longrightarrow} 2 \mathrm{Br}^{-}+2 \mathrm{Ag}^{0}+2 \mathrm{H}_{2} \mathrm{O}
$$

Fig. 3(c) shows the $\mathrm{pH}$ of growth solution with different concentrations of $\mathrm{NaOH}$, and the changes in $\mathrm{pH}(\Delta \mathrm{pH}$, the difference between the growth solutions aged for $2 \mathrm{~h}$ (marked 2) and aged for $0 \mathrm{~h}$ (marked 1)). When the concentration of $\mathrm{NaOH}$ was not more than $7.6 \mathrm{mM}$, the growth solution was acidic and there was a minor difference between the $\mathrm{pH}$ of the solution aged for $2 \mathrm{~h}$ and the unaged solution $(\Delta \mathrm{pH} \approx 0)$, representing that the reduction of $\mathrm{Ag}^{+}$did not happen or the reduction rate of $\mathrm{Ag}^{+}$was very slow. When the concentration of $\mathrm{NaOH}$ was over $7.6 \mathrm{mM}$, the $\mathrm{pH}$ of the growth solution aged for $2 \mathrm{~h}$ is lower than that of the unaged solution $(\Delta \mathrm{pH}<0)$, which indicated that the reduction reaction still progressed and $\mathrm{Ag}^{+}$was reduced to form AgNPs.

Apart from the $\mathrm{NaOH}$ consumption caused by the $\mathrm{Ag}^{+}$involved in the reaction, more $\mathrm{NaOH}$ may be consumed by the reduction of Vc anions in the aging process of growth solution exposed to the atmosphere. Generally, Vc is easily decomposed under alkaline conditions to produce 2-3-diketogulonic acid. Then, this compound will further oxidize to arabitic acid and oxalic acid. In this process, the consumption of $\mathrm{OH}^{-}$in the growth solution increased and the $\mathrm{pH}$ value of the growth solution decreased. Furthermore, some $\mathrm{OH}^{-}$ions would be adsorbed on the surface of CTAB micelles by replacing $\mathrm{Br}^{-}$ions to further decrease the $\mathrm{pH}$ of the growth solution in its aging process.

The inflection point of $\Delta \mathrm{pH}$ is at the $\mathrm{NaOH}$ concentration range of 15-20 $\mathrm{mM}$, which corresponds to the sign of the reduction rate of $\mathrm{Ag}^{+}$. It is also the key point of the shape transformation from triangular silver nanoplates to silver nanorods. It was reported that the $\mathrm{pH}$ value of the growth solution has a strong influence on the redox potential of $\mathrm{Vc}^{48}$ The first ionization and second ionization of $\mathrm{Vc}$, related to the $\mathrm{pH}$ values of the solution and thus the reaction activity of $\mathrm{Vc}$, is different under different $\mathrm{pH}$ solutions. The reduction rate of $\mathrm{Ag}^{+}$ by $\mathrm{Vc}$ is very slow at $\mathrm{pH} 3.5-5.0$, while the reduction rate is higher when the $\mathrm{pH}$ value of the solution is higher. The reduction rate of $\mathrm{Ag}^{+}$by $\mathrm{Vc}$ was so slow that almost no nanoparticles were formed at $\mathrm{pH}<5$ in the beginning of the reaction. When the $\mathrm{pH}$ was between 8.2 and 10.8 , the reduction rate of $\mathrm{Ag}^{+}$increased to form $\mathrm{Ag}^{0}$ for the growth of silver seeds capped with citrate- $\mathrm{CTA}^{+}$to form triangular silver nanoplates, which may be caused by the selective adsorption on the special crystal surface of the seeds. When the $\mathrm{pH}$ was higher than 11.5 , the reduction rate of $\mathrm{Ag}^{+}$further increased to generate more $\mathrm{Ag}^{0}$, the silver seeds grew to form silver nanorods under the coating and guidance effects of the rod-like CTAB micelles. However, a large amount of $\mathrm{Ag}^{0}$ produced at the $\mathrm{pH}$ over $\mathbf{1 1 . 5}$ easily aggregated to shapes resembling nanospheres, which decreased the aspect ratio of the formed nanorods. The above results indicated that the varied redox potential of $\mathrm{Vc}$ caused by different $\mathrm{pH}$ in the growth solution of AgNPs plays a key role in controlling the morphology of AgNPs and the side length of silver nanoplates or aspect ratio of silver nanorods.

\subsection{The influence of the aging time of growth solution on the morphology of silver nanoplates}

Fig. 4 shows the influence of the aging time of the growth solution on the morphology of the silver nanoplates. The UV-vis spectra (Fig. 4(a)) show three typical absorption peaks of the triangular silver nanoplates. Both the $\lambda_{\max }$ and the intensity of the absorption peak in the long-wave direction decreases with an increase in aging times, indicating that the side length of the triangular nanoplates decreases with aging time..$^{32,44,45}$ Furthermore, the corner sharpness of the truncated triangular nanoplates in growth solution is related to the blue shift in the $\lambda_{\max }$ of the absorption peak in the long-wave direction. ${ }^{\mathbf{1 9 , 3 2 , 4 4 , 4 5}}$ In the UV-vis spectra of growth solution with different aging times (Fig. 4(a)), a blue shift of $\lambda_{\max }$ in the long-wave direction could be clearly observed. Thus, the as-prepared triangular nanoplates should be truncated triangular nanoplates.

Fig. 4(b) shows the photos of growth solutions prepared by the seeds aged for different times (see the horizontal axis) and growth solution at different aging times (see the vertical axis). The color of the fresh growth solution (aged for $0 \mathrm{~d}$ ) changed from yellow to red, purple, and blue, while the aging time of the seed used for preparing AgNPs was from 0, 10, 20, and over $30 \mathrm{~min}$. The changes in color of the growth solution indicated that we could prepare AgNPs with different morphologies by using seeds aged for different times in the proposed improved method, which is consistent with the deduction from the spectral results shown in Fig. 2(a) and (b).

The color of growth solution faded with aging time (as shown the vertical axis in Fig. 4(b)). For example, the color of the 

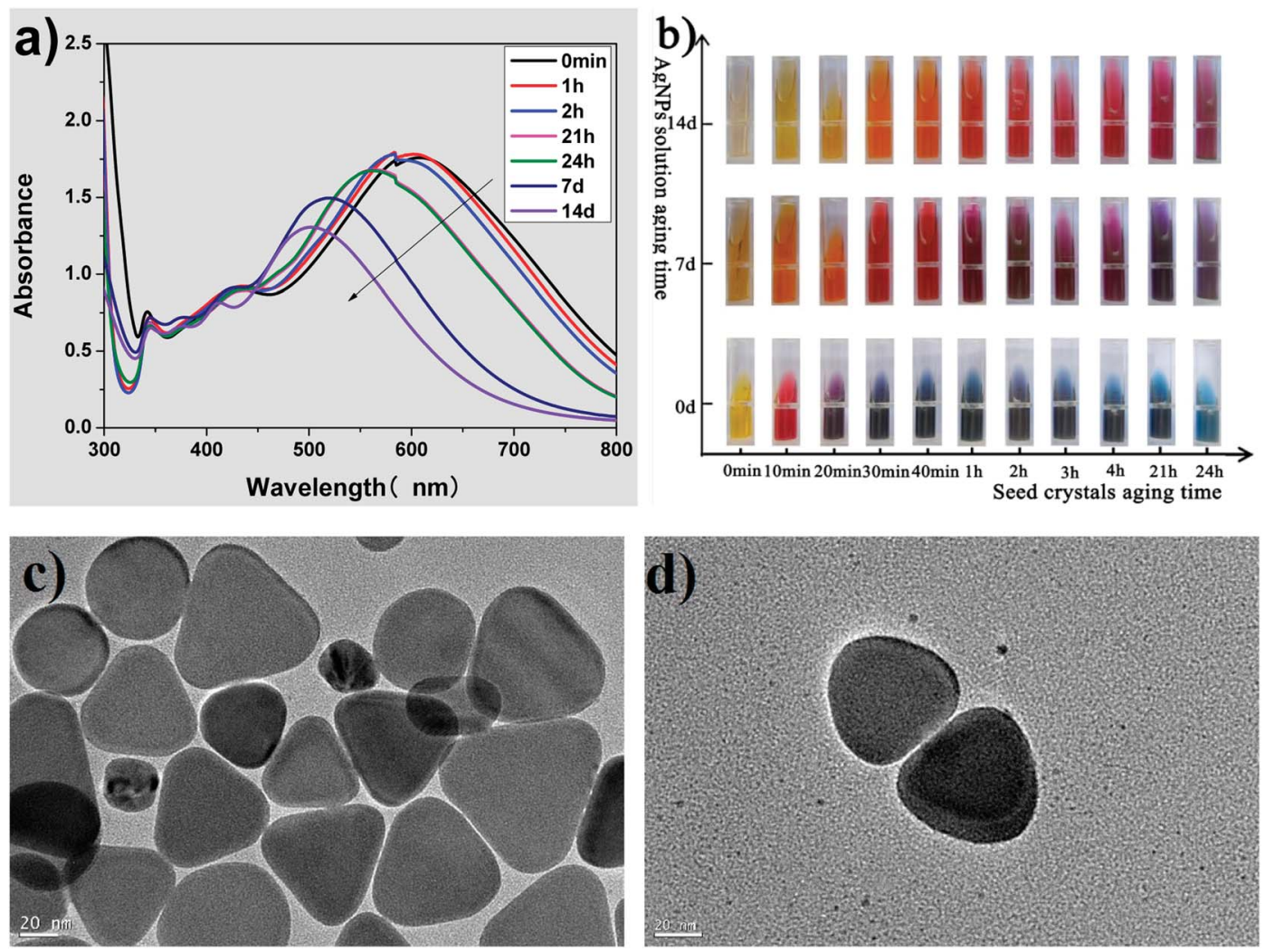

Fig. 4 The influence of aging time of growth solution on the morphology of silver nanoplates: (a) UV-vis spectra of growth solution aged for different times; (b) photos of growth solution at different aging times (see the vertical axis) and photos of growth solution prepared respectively by the seed at different aging times (see the horizontal axis); TEM image of silver nanoplates prepared by the seed aged for $3 \mathrm{~h}$ : (c) the growth solution aging time was $24 \mathrm{~h}$ and (d) the growth solution aging time was $3 \mathrm{~d}$.

growth solution prepared by the seed aged for $1 \mathrm{~h}$ was blue, purple and orange-red, while the growth solution was aged for 0,7 and $14 \mathrm{~d}$, respectively. The changes in the color implied that the $\lambda_{\max }$ of the aged collosol blue-shifted in the long-wave direction and thus, the side length of the triangular nanoplates decreased with the aging time,$^{32,44,45}$ which is consistent with the UV-vis spectra of growth solution aged for different times, as shown in Fig. 4(a).

Fig. 4(c) shows the TEM images of triangular nanoplates prepared by seeds whose aging time was $3 \mathrm{~h}$ and the aging time of growth solution was $24 \mathrm{~h}$. The as-prepared AgNPs are perfectly truncated triangular nanoplates with few particles resembling nanospheres, although there is some difference in the side length among the triangular nanoplates. For comparison, the rounded corners of triangular AgNPs are clearly observed in Fig. 4(d) while the growth solution was aged for 3 days. Due to the oxidation etching effect of $\mathrm{Br}^{-} / \mathrm{O}_{2},{ }^{17-19}$ the corner sharpness of the truncated triangular nanoplates further decreased, and the nanoplates transformed into quasi-circular triangular nanoplates with aging time.

\subsection{A possible mechanism for the formation of seed and triangular silver nanoplates in CTAB solution}

In our improved seed preparation method, a moderate amount of CTAB was added for the formation of silver seed and thus, the precursors could be $\mathrm{AgBr}$ and $\mathrm{AgL}$ (L represents for sodium citrate). In order to explore the relative amounts of $\mathrm{AgBr}$ and AgL, a theoretical calculation was performed, and the calculation result showed that the main component in the seed colloidal solution was AgBr but not AgL. This result was also preliminarily verified by an experiment shown in ESI (Section 1, Fig. S1†); hence, we proposed that the AgBr could be formed in the solution.

After the addition of $\mathrm{AgNO}_{3}$ and sodium citrate, $\mathrm{Ag}^{+}$and $\mathrm{Br}^{-}$ derived from CTAB formed AgBr. As a result, CTAB was adsorbed on the edge of $\mathrm{AgBr}$ particles to form CTAB spherical micelles, and the free $\mathrm{CTA}^{+}\left(\mathrm{CTA}^{+}\right.$is cetyltrimethyl ammonium) was complexed with citrate anions to form the citrate-CTA ${ }^{+}$ complex..$^{50-52}$ When $\mathrm{KBH}_{4}$ was added with vigorous stirring, $\mathrm{AgBr}$ particles solubilized by CTAB-spherical micelles were reduced to silver atoms $\left(\mathrm{Ag}^{0}\right)$ (reaction (2)).

$$
\mathrm{BH}_{4}^{-}+8 \mathrm{AgBr}+8 \mathrm{OH}^{-}=8 \mathrm{Ag}^{0}+\mathrm{BO}_{2}^{-}+8 \mathrm{Br}^{-}+6 \mathrm{H}_{2} \mathrm{O}
$$

Owing to the oxidative etching effect of the $\mathrm{Br}^{-} / \mathrm{O}_{2}$ pair, ${ }^{17-19}$ the formed $\mathrm{Ag}^{0}$ was oxidized to release $\mathrm{Ag}^{+}$to form $\mathrm{AgBr}$ solubilized by the spherical micelles (reaction (3)). The circular reactions ((2) and (3)) continued until $\mathrm{KBH}_{4}$ was completely consumed.

$$
2 \mathrm{Ag}^{0}+1 / 2 \mathrm{O}_{2}+\mathrm{H}_{2} \mathrm{O}=2 \mathrm{Ag}^{+}+2 \mathrm{OH}^{-}
$$

Based on the result shown in Fig. 1(a), the seed nanocrystals are unstable at the CTAB concentration of less than $0.96 \mathrm{mM}$. 


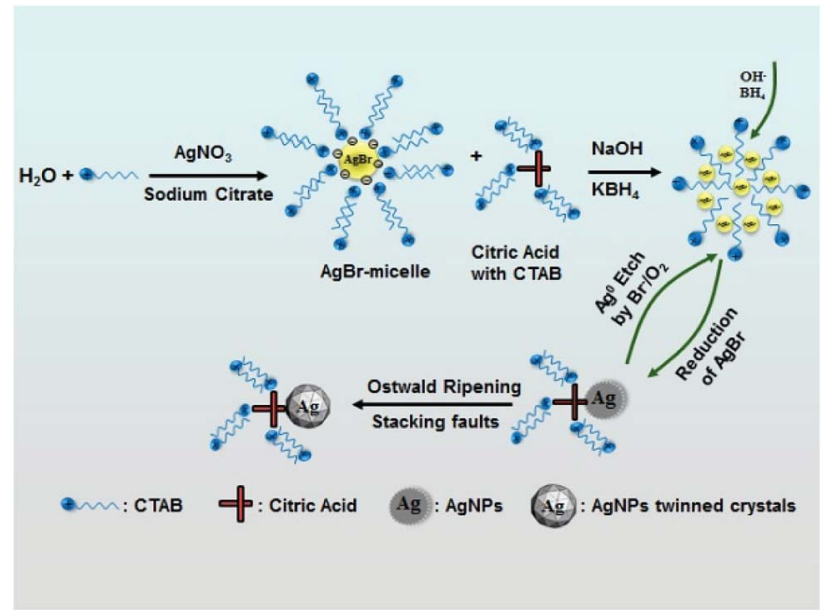

Fig. 5 A schematic illustrating the formation of silver seed in the solution containing CTAB.

The seeds could grow to triangular-like AgNPs with different sizes only at the concentration of more than $0.96 \mathrm{mM}$ (as shown in Fig. 1(b)), which was different from the citrate-coated silver seed prepared by the conventional method. ${ }^{38}$ In this case, the surface of silver seed nanocrystals should be adsorbed by the citrate-CTA $^{+}$complex, due to which the stability of the seed nanocrystals improved significantly. Based on the above discussion, a possible mechanism for the formation of silver seed in the solution containing CTAB is proposed, as shown in Fig. 5.

Fig. 6 shows the FTIR spectra of triangular silver nanoplates obtained by the silver seed capped with citrate-CTA ${ }^{+}$and some references. In the spectrum of CTAB (Line 1), the peaks at 2849 and $2918 \mathrm{~cm}^{-1}$ are attributed to $-\mathrm{CH}_{2}$ symmetric and asymmetric stretching vibrations and the peaks at about $1487 \mathrm{~cm}^{-1}$ are related to ${ }^{+} \mathrm{N}-\mathrm{CH}_{3}$ stretching vibrations of the CTAB headgroup. In the spectrum of citrate (Line 4), the peaks at 3452 and $3271 \mathrm{~cm}^{-1}$ are attributed to the stretching vibrations of free

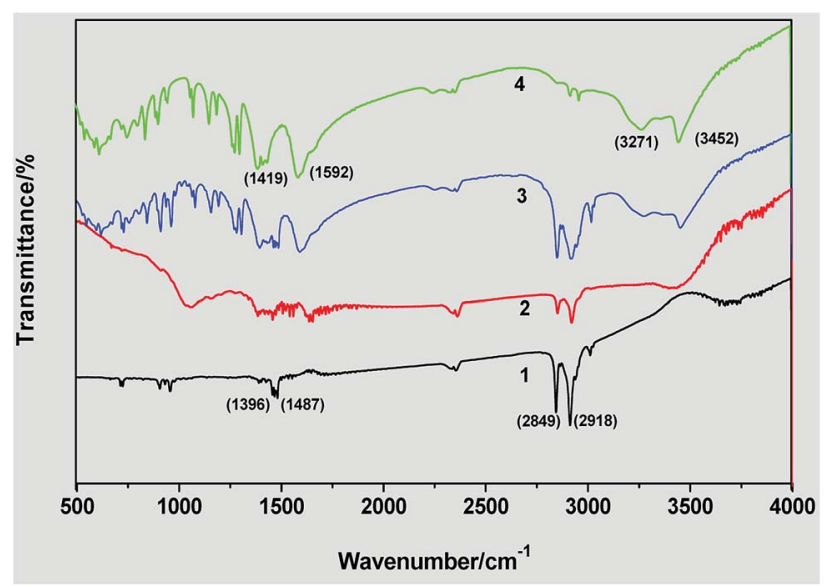

Fig. 6 FTIR spectra of triangular silver nanoplates by the silver seed capped with citrate-CTA : (1) CTAB, (2) sample of silver nanoplates, (3) mixture of $C T A B$ and citrate, and (4) citrate.

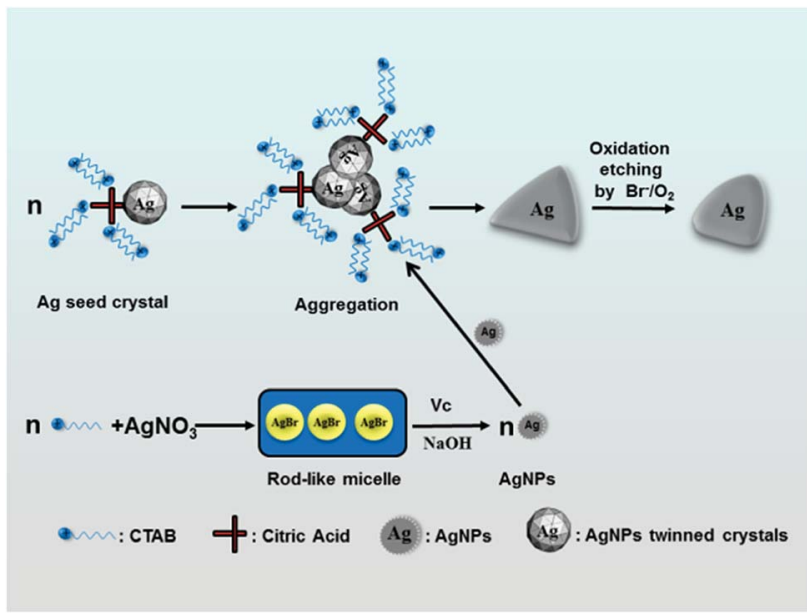

Fig. 7 A schematic illustrating the formation of triangular silver nanoplates by the orientated stacking and growth of the seed.

$-\mathrm{OH}$ and associated $-\mathrm{OH}$ and the peaks at 1592 and about $1419 \mathrm{~cm}^{-1}$ are attributed to the stretching vibrations of $-\mathrm{C}=\mathrm{O}$ and symmetric and asymmetric stretching vibrations of $-\mathrm{CH}_{2}$.

The spectrum of the obtained AgNPs (Line 2) is similar to that of the mixture of CTAB and citrate (Line 3) in the above main absorption peaks, implying that the formation of triangular silver nanoplates is due to the mixed adsorption of CTAB and citrate. As the CTAB molecules capped AgNPs via their headgroups and citrate molecules capped AgNPs via their carboxylate ions, the absorption peaks of ${ }^{+} \mathrm{N}-\mathrm{CH}_{3}$ in CTAB and the absorption peak of $-\mathrm{C}=\mathrm{O}$ in citrate has considerable variation in their corresponding absorption peaks in the spectrum of AgNPs samples.

A possible mechanism for the formation of triangular silver nanoplates is outlined in Fig. 7. With the addition of $\mathrm{AgNO}_{3}$, the $\mathrm{AgBr}$ colloidal particles formed by precipitation reaction between $\mathrm{Ag}^{+}$and $\mathrm{Br}^{-}$are mainly dissolved into the rod-like micelles. On adding silver seeds, $\mathrm{Vc}$ and $\mathrm{NaOH}$ into the growth solution with vigorous stirring, $\mathrm{AgBr}$ is reduced to $\mathrm{Ag}^{0}$ by Vc. Silver seed crystals may aggregate or stack along with the special crystal planes with no or minor adsorption to form triangle-like agglomerates with the help of the selective adsorption of the citrate-CTA ${ }^{+}$complex.

Along with the reduction of $\mathrm{Ag}^{+}$ions, many $\mathrm{Ag}^{0}$ are brought to selectively form triangular silver nanoplates on the (111) crystal surface of the seed, ${ }^{50-52}$ and the triangle-like agglomerates grow into triangle-like nanoparticles. With the increase in aging time of growth solution, triangular silver nanoplates are passivated to form truncated triangular nanoplates under the condition of the oxidative etching of $\mathrm{Br}^{-} / \mathrm{O}_{2}$ pair. ${ }^{17-19}$

\section{Conclusion}

In this paper, we have provided a simple but effective technique to synthesize silver nanotriangles by using an improved silver seed. Compared to the preparation of silver nanoparticles (AgNPs) by a typical seed-mediated method, the seeds prepared 
by our method have a longer use time, and various AgNPs, particularly silver nanotriangles with different particle sizes can be easily prepared by the silver seed capped with citrate-CTA ${ }^{+}$ instead of silver nanorods. The morphology and size of the obtained AgNPs are highly dependent on the concentration of $\mathrm{CTAB}$ in seed collosol, the aging time of the seed and the $\mathrm{pH}$ value of the growth solution.

The inflection point of the use time of the seed collosol is about $20 \mathrm{~min}$, the inflection point of the concentration of CTAB in seed is between 0.96 and $1.40 \mathrm{mM}$, and the inflection point of $\mathrm{NaOH}$ concentration in the growth solution is about $15 \mathrm{mM}$, corresponding to the key points of the shape transformation. The truncated triangular nanoplates were formed at following conditions: the concentration of CTAB in seed was between 0.96 and $1.40 \mathrm{mM}$, the seed was aged for over $20 \mathrm{~min}$ and could be used for $24 \mathrm{~h}$ or longer, and the shape transformation from triangular silver nanoplates to silver nanorods occurred at the $\mathrm{NaOH}$ concentration of 15-20 mM (pH 10.8-11.3). Based on the limited experimental results and IR analysis, a possible mechanism was preliminarily proposed to explain the formation of the seed crystals and truncated triangular AgNPs in our improved system.

\section{Conflicts of interest}

The authors declare that they have no conflict of interest.

\section{Acknowledgements}

This project was supported by the National Natural Science Foundation of China (Grant No. 81071254) and the Natural Science Foundation of Guangdong Province, China (Grant No. 10451051501004706).

\section{References}

1 A. D'Agostino, A. Taglietti, R. Desando, M. Bini, M. Patrini, G. Dacarro, L. Cucca, P. Pallavicini and P. Grisoli, Nanomaterials, 2017, 7, 16.

2 H. Yu, L. L. Li and Y. J. Zhang, Scr. Mater., 2012, 66, 931-934.

3 S. Hamedi, S. A. Shojaosadati and A. Mohammadi, J. Photochem. Photobiol., B, 2017, 167, 36-44.

4 Q. Tian, X. J. Yu, L. F. Zhang and D. M. Yu, J. Colloid Interface Sci., 2017, 491, 294-304.

5 M. Liang, G. Zhang, Y. J. Feng, R. L. Li, P. Hou, J. S. Zhang and J. M. Wang, J. Mater. Sci., 2018, 53, 1568-1579.

6 X. P. Zeng, Q. Wang, H. Wang and Y. J. Yang, J. Mater. Sci., 2017, 52, 8391-8400.

7 C. H. Deng, J. L. Gong, P. Zhang, G. M. Zeng, B. Song and H. Y. Liu, J. Colloid Interface Sci., 2017, 488, 26-38.

8 S. Tavakol, E. Hoveizi, S. Kharrazi, B. Tavakol, S. Karimi and S. M. R. Sorkhabadi, Artif. Cells, Nanomed., Biotechnol., 2017, 45, 817-823.

9 G. Hu, Y. Cai, Z. Tu, J. Luo, X. Qiao, Q. Chen and W. Zhang, RSC Adv., 2015, 5, 82050-82055.

10 G. S. Hu, W. X. Jin, Q. Y. Chen, Y. C. Cai, Q. H. Zhu and W. Z. Zhang, Appl. Phys. A: Mater. Sci. Process., 2016, 122, 7.
11 S. Agnihotri, S. Mukherji and S. Mukherji, RSC Adv., 2014, 4, 3974-3983.

12 M. Nabil and P. Zunino, R. Soc. Open Sci., 2016, 3, 15.

13 G. S. Hu, G. R. Liang, W. Zhang, W. X. Jin, Y. Zhang, Q. Y. Chen, Y. C. Cai and W. Z. Zhang, J. Mater. Sci., 2018, 53, 4768-4780.

14 S. Adepu and M. Khandelwal, J. Mater. Sci., 2018, 53, 15961609.

15 M. Kumar, R. Bala, V. S. Gondil, S. K. Pandey, S. Chhibber, D. V. S. Jain, R. K. Sharma and N. Wangoo, J. Mater. Sci., 2017, 52, 8568-8575.

16 P. Paril, J. Baar, P. Cermak, P. Rademacher, R. Prucek, M. Sivera and A. Panacek, J. Mater. Sci., 2017, 52, 2720-2729.

17 Y. Wang, D. Wan, S. Xie, X. Xia, C. Z. Huang and Y. Xia, ACS Nano, 2013, 7, 4586-4594.

18 H. Y. Liang, W. Z. Wang, Y. Z. Huang, S. P. Zhang, H. Wei and H. X. Xu, J. Phys. Chem. C, 2010, 114, 7427-7431.

19 M. Rycenga, C. M. Cobley, J. Zeng, W. Y. Li, C. H. Moran, Q. Zhang, D. Qin and Y. N. Xia, Chem. Rev., 2011, 111, 3669-3712.

20 S. Zhou, J. H. Li, K. D. Gilroy, J. Tao, C. L. Zhu, X. Yang, X. J. Sun and Y. N. Xia, ACS Nano, 2016, 10, 9861-9870.

21 A. D'Agostino, A. Taglietti, P. Grisoli, G. Dacarro, L. Cucca, M. Patrini and P. Pallavicini, RSC Adv., 2016, 6, 70414-70423.

22 Y. K. Kim and D. H. Min, Surface confined successive growth of silver nanoplates on a solid substrate with tunable surface plasmon resonance, RSC Adv., 2014, 4(14), 6950-6956.

23 X. C. Jiang, Q. H. Zeng and A. B. Yu, Nanotechnology, 2006, 17, 4929-4935.

24 M. Z. Liu, M. Leng, C. Yu, X. Wang and C. Wang, Nano Res., 2010, 3, 843-851.

25 S. N. Chen, X. Li, S. Han, J. H. Liu and Y. Y. Zhao, RSC Adv., 2015, 5, 99914-99919.

26 A. A. Furletov, V. V. Apyari, A. V. Garshev, S. G. Dmitrienko and Y. A. Zolotov, J. Anal. Chem., 2017, 72, 1203-1207.

27 V. V. Apyari, M. O. Gorbunova, A. V. Shevchenko, A. A. Furletov, P. A. Volkov, A. V. Garshev, S. G. Dmitrienko and Y. A. Zolotov, Talanta, 2018, 176, 406-411.

28 M. O. Gorbunova, A. V. Shevchenko, V. V. Apyari, A. A. Furletov, P. A. Volkov, A. V. Garshev and S. G. Dmitrienko, Sens. Actuators, B, 2018, 256, 699-705.

29 S. B. Liu, H. B. Tao, L. Zeng, Q. Liu, Z. G. Xu, Q. X. Liu and J. L. Luo, J. Am. Chem. Soc., 2017, 139, 2160-2163.

30 G. W. Yang and H. L. Li, Mater. Lett., 2008, 62, 2189-2191.

31 M. H. Kim, D. K. Yoon and S. H. Im, $R S C A d v$., 2015, 5, 14266-14272.

32 R. Jin, Y. Cao, C. A. Mirkin, K. L. Kelly, G. C. Schatz and J. G. Zheng, Science, 2001, 294, 1901-1903.

33 L. C. Yang, Y. S. Lai, C. M. Tsai, Y. T. Kong, C. I. Lee and C. L. Huang, J. Phys. Chem. C, 2012, 116, 24292-24300.

34 S. H. Ciou, Y. W. Cao, H. C. Huang, D. Y. Su and C. L. Huang, J. Phys. Chem. C, 2009, 113, 9520-9525.

35 J. An, B. Tang, X. L. Zheng, J. Zhou, F. X. Dong, S. P. Xu, Y. Wang, B. Zhao and W. Q. Xu, J. Phys. Chem. C, 2008, 112, 15176-15182.

36 B. Tang, L. Sun, J. L. Li, M. W. Zhang and X. A. Wang, Chem. Eng. J., 2015, 260, 99-106. 
37 X. L. Zheng, Y. S. Peng, J. R. Lombardi, X. Q. Cui and W. T. Zheng, Colloid Polym. Sci., 2016, 294, 911-916.

38 N. R. Jana, L. Gearheart and C. J. Murphy, Chem. Commun., 2001, 617-618.

39 Q. Zhang and Y. D. Yin, Chem. Commun., 2013, 49, 215-217. 40 B. Nikoobakht and M. A. El-Sayed, Chem. Mater., 2003, 15, 1957-1962.

41 T. S. Sabir, L. K. Rowland, J. R. Milligan, D. Yan, A. W. Aruni, Q. Chen, D. S. Boskovic, R. S. Kurti and C. C. Perry, Langmuir, 2013, 29, 3903-3911.

42 X. H. Cui, S. Z. Mao, M. L. Liu, H. Z. Yuan and Y. R. Du, Langmuir, 2008, 24, 10771-10775.

43 W. Z. Zhang, X. L. Qiao, X. L. Qiu, Q. Y. Chen, Y. C. Cai and H. Q. Chen, Curr. Nanosci., 2013, 9, 753-758.

44 J. E. Millstone, S. J. Hurst, G. S. Metraux, J. I. Cutler and C. A. Mirkin, Small, 2009, 5, 646-664.
45 R. Jin, Y. C. Cao, E. Hao, G. S. Metraux, G. C. Schatz and C. A. Mirkin, Nature, 2003, 425, 487-490.

46 B. J. Wiley, S. H. Im, Z. Y. Li, J. McLellan, A. Siekkinen and Y. N. Xia, J. Phys. Chem. B, 2006, 110, 15666-15675.

47 W. Z. Zhang, X. L. Qiao, Q. Y. Chen, Y. C. Cai and H. Q. Chen, Appl. Surf. Sci., 2012, 258, 5909-5913.

48 W. Z. Zhang, W. Zhang, X. L. Qiao, X. L. Qiu, Q. Y. Chen and Y. C. Cai, Sci. Adv. Mater., 2014, 6, 304-311.

49 N. R. Jana, Small, 2005, 1, 875-882.

50 J. Zeng, J. Tao, W. Li, J. Grant, P. Wang, Y. Zhu and Y. Xia, Chem. - Asian J., 2011, 6, 376-379.

51 M. Tsuji, S. Gomi, Y. Maeda, M. Matsunaga, S. Hikino, K. Uto, T. Tsuji and H. Kawazumi, Langmuir, 2012, 28, 8845-8861.

52 Q. Zhang, N. Li, J. Goebl, Z. Lu and Y. Yin, J. Am. Chem. Soc., 2011, 133, 18931-18939. 